\title{
Comparison of Three Analytical Methods for the Determination of Quinolones in Pig Muscle Samples
}

\author{
Inmaculada Jiménez-Díaz ${ }^{1}$, María Pilar Hermo², Oscar Ballesteros ${ }^{* 1}$, Alberto \\ Zafra-Gómez ${ }^{1}$, Dolores Barrón ${ }^{2}$, José Barbosa ${ }^{2}$, Alberto Navalón ${ }^{1}$ \\ ${ }^{1}$ Research Group of Analytical Chemistry and Life Sciences, Department of Analytical \\ Chemistry, Campus of Fuentenueva, University of Granada, E-18071 Granada, Spain \\ ${ }^{2}$ Research Group of Bioanalysis, Department of Analytical Chemistry, University of Barcelona, \\ Avda. Diagonal 647, E-08028 Barcelona, Spain
}

Abstract This work presents a comparison between three analytical methods developed for the simultaneous determination of eight quinolones regulated by the European Union (marbofloxacin, ciprofloxacin, danofloxacin, enrofloxacin, difloxacin, sarafloxacin, oxolinic acid and flumequine) in pig muscle, using liquid chromatography with fluorescence detection (LC-FD), liquid chromatography-mass spectrometry (LCMS) and liquid chromatography-tandem mass spectrometry (LC-MS/MS). The procedures involve an extraction of the quinolones from the tissues, a step for clean-up and preconcentration of the analytes by solid-phase extraction (SPE) and a subsequent liquid chromatographic analysis. The limits of detection of the methods ranged from 0.1 to $2.1 \mathrm{ng} \mathrm{g}^{-1}$ using LC-FD, from 0.3 to 1.8 using LC-MS and from 0.2 to 0.3 using LCMS/MS, while inter- and intra-day variability was under $15 \%$ in all cases. Most of those data are notably lower than the maximum residue limits (MRL) established by the European Union for quinolones in pig tissues. The methods have been applied for the determination of quinolones in six different commercial pig muscle samples purchased in different supermarkets located in the city of Granada (South-East Spain).

Keywords Liquid chromatography - Fluorescence detection - Mass spectrometry detection · Quinolones · Pig muscle · Methods comparison

\footnotetext{
*Corresponding author: Tel.: +34 958243326; fax: +34 958243328.

E-mail address: oballest@ugr.es (O. Ballesteros)
} 


\section{Introduction}

46 The research in the field of contamination in foods has extended in the last years beyond

47 classical contaminants-pesticides, biocides, polyaromatic hydrocarbons, dioxins or polychlorinated biphenyls-to other compounds such as pharmaceuticals or personal care products [1]. Since pharmaceuticals are produced and applied with the aim of being biologically active and stimulate a physiological response in human and animals even at low concentrations, there is a growing concern in relation to these substances and their recognition as contaminants, mainly due to the adverse effects that their wide use and disposal have on human health [2]. European consumption of pharmaceuticals is known to be increasing on a yearly basis, and today more than 5000 products are being used as painkillers, contraceptives, tranquilizers, lipid regulators, beta-blockers or antibiotics [1]. Antibiotics and their degradation metabolites rank among the most used drugs in human and veterinary medicine. Resistance to antibiotics and other anti-infective agents constitutes a major threat to public health and ought to be recognized as such more widely than it is currently. Therefore, the European Union (EU) recommends the prudent use of antimicrobial agent in human medicine.

One of the most important groups of antibiotics is quinolones. They are a family of highly potent antibiotics with a broad spectrum of activity against both Gramnegative and Gram-positive pathogens. They are widely used in human and veterinary medicine in the treatment of infections and represent an expanding class of broadspectrum antibacterials [3]. Quinolones have become an integral part of the livestock production industry and can be used therapeutically to treat disease or to prevent it as well as for promoting growth [4]. Their use in veterinary applications can result in the appearance of residues of the compounds and metabolites in edible animal meats and may give rise to public health concerns, including development of resistant bacterial strains, toxic effects or allergic hypersensitivity [5]. Some international organizations such as the World Health Organization (WHO) have recommended a higher attention and control in the use of antimicrobial growth promoters that belong to an antimicrobial class used in humans. The EU agreed to reduce the use of all antimicrobial growth promoters from 2002. To ensure safety, it has been established maximum residue limits (MRLs) for veterinary drugs in those animal tissues that enter the human food chain [6- 
77 other tissues as kidney or liver. So, the MRL in pig muscle for enrofloxacin plus 78 ciprofloxacin, danofloxacin and oxolinic acid are fixed at $100 \mathrm{ng} \mathrm{g}^{-1}$, for marbofloxacin 79 and flumequine at 150 and $200 \mathrm{ng} \mathrm{g}^{-1}$ respectively, while for difloxacin the MRL is 400 $80 \mathrm{ng} \mathrm{g}^{-1}$. The MRL of sarafloxacin, major metabolite of difloxacin is not yet established. 81 Therefore, more analytical methodology is demanded to quantify and confirm the 82 identity of these compounds in food-producing animal. In the scientific literature, some 83 analytical methodologies have been described for the determination of fluoroquinolone 84 residues in animal derived foods. Given the complexity of these samples, the majority 85 of these methodologies are based in the use of liquid chromatography (LC) with ultraviolet (UV) [10], fluorescence (FD) [11] or mass spectrometric (MS) detection [12] after sample clean-up by SPE [10-12]. Owing to its specificity, mass spectrometry is the most powerful confirmatory technique; however, it is expensive and thus not available to all laboratories. In the case of fluorescent drugs, as quinolones, because of its selectivity and sensitivity, FD is a very good detection approach.

The main objective of this work is to compare the quality control parameters of three different analytical methodologies developed using LC-FD, LC-MS or LCMS/MS for the determination of quinolones in pig muscle samples in order to provide the method that has the best analytical characteristics. The three analytical methodologies were satisfactorily applied for the quantification of compounds in samples picked up at different supermarkets of Granada (Spain).

\section{Experimental}

Chemical and reagents

103 Pure quinolone standards were purchased from different pharmaceutical companies.

104 Ciprofloxacin (CIP) from Ipsen Pharma (Barcelona, Spain); danofloxacin (DAN) from 105 Pfizer (Karlsruhe, Germany); difloxacin (DIF) and sarafloxacin (SAR) from Abbott 106 (Madrid, Spain); enrofloxacina (ENR) from Cenavisa (Tarragona, Spain); flumequine 107 (FLU), norfloxacina (NOR) and oxolinic acid (OXO) form Sigma-Aldrich (Madrid, 108 Spain) and marbofloxacin (MAR) from Vetoquinol (Lure, France). 
Acetonitrile, MeCN (LC-grade), $o$-phosphoric and citric acids were obtained

110 from Panreac (Barcelona, Spain). Methanol, ethanol, hexane, ammonia, formic acid,

111 trifluoroacetic acid and $m$-phosphoric acid were supplied by Merck (Darmstadt,

112 Germany). Isolute ENV+ $(200 \mathrm{mg} / 3 \mathrm{~mL})$ solid-phase extraction (SPE) adsorbent

113 cartridges were purchased from Isolute Sorbent Technologies (Mid Glamorgan, UK).

115 Instrumentation and software

117 LC-FD analysis were performed using an HP Agilent Technologies (Palo Alto, CA,

118 USA) 1100 series liquid chromatography system with fluorescence detector connected

119 on-line. ChemStation for LC 3D software (Agilent) was used for instrument control and

120 for data acquisition and analysis. LC-MS and LC-MS/MS analysis were performed

121 using an API 3000 (Applied Biosystems, Foster City, CA, USA) triple quadrupole mass

122 spectrometer system. In order to obtain data, the Analyst 1.4 software was used.

All pH measurements were made with a Crison (Crison Instruments S.A,

124 Barcelona, Spain) combined glass- $\mathrm{Ag} / \mathrm{AgCl}(\mathrm{KCl} 3 \mathrm{M})$ electrode using a previously calibrated Crison 2000 digital pH-meter. A Branson digital sonifier (Danbury, CT, USA) and a Hettich Universal 32 centrifuge (Tuttlingen, Germany) were also used. SPE was performed on a Supelco (Madrid, Spain) vacuum manifold for 12 columns connected to a Supelco vacuum tank and to a vacuum pump. Statgraphics software was used for statistical and regression analysis.

Preparation of standard and stock solutions

133 For LC-MS and LC-MS/MS analyses individual stock solutions of CIP, DAN, DIF, 134 ENR, MAR, NOR, and SAR $\left(100 \mu \mathrm{g} \mathrm{mL}^{-1}\right)$, were prepared in $50 \mathrm{mM}$ acetic acid aqueous solution. FLU and OXO $\left(100 \mu \mathrm{g} \mathrm{mL}^{-1}\right)$ were prepared in MeCN. Individual working solutions were prepared by diluting the initial standard solutions with MeCN.

For LC-FD analysis, individual stock solutions of CIP, DAN, DIF, ENR, MAR,

138 NOR and SAR $\left(100 \mu \mathrm{g} \mathrm{mL}^{-1}\right)$ were prepared in ethanol $(99.9 \% v / v)$. Individual stock solutions of FLU and OXO $\left(100 \mu \mathrm{g} \mathrm{mL}^{-1}\right)$ were prepared in MeCN. Individual working

140 solutions were prepared by diluting suitably with a MeCN-water mixture $(12: 88, v / v)$.

141 All solutions were stored at $4{ }^{\circ} \mathrm{C}$ in the dark for not longer than 2 months. 
145 Fortified samples were prepared by spiking $5 \mathrm{~g}$ (accurately weighed) of minced blank

146 pig muscle adding the adequate volumes of working solutions of studied quinolones and 147 norfloxacin -a forbidden veterinary quinolone- used as surrogate. Before sample

148 treatment and analysis, all samples were allowed to stand in the dark for $20 \mathrm{~min}$ at room

149 temperature to permit the total interaction between the antibiotics and tissues. In order

150 to evaluate recoveries, spiked samples in the same range of concentration were prepared 151 and compared with samples spiked after the SPE procedure and that were considered 152 the $100 \%$ of recovery.

154 Basic procedure

156 Two methods previously published by the authors were followed for sample treatment

$157[11,12]$. The procedures involve an extraction of the quinolones from the tissues by 158 shaking, a clean-up and preconcentration step by solid-phase extraction (SPE) and a subsequent liquid chromatographic analysis.

\section{Results and discussion}

164 Validation of the methods

Analytical performance

168 For LC-MS/MS calibration, the studied concentration levels ranged from 0.5 to 100.0 $169 \mathrm{ng} \mathrm{g}^{-1}$; for LC LC-MS from 5.0 to 100.0 and for LC-FD from 5.0 to 50.0. In all cases 170 each level of concentration was made in duplicate. Calibration curves were constructed 171 using analyte/surrogate peak area ratio versus concentration of analyte. Norfloxacin 172 (400 $\mathrm{ng} \mathrm{g}^{-1}$ for LC-MS/MS and LC-MS calibration and $20 \mathrm{ng} \mathrm{g}^{-1}$ for LC-FD 173 calibration) was used as surrogate. The lack-of fit test was used to check the linearity of 174 the calibration graphs according to the Analytical Methods Committee [13]. Table 1 175 shows the calibration parameters obtained (intercepts and slopes). 
180 Validation of the methods was performed according to the US Food and Drugs 181 Administration (FDA) guideline for bioanalytical assay procedure [13] in terms of 182 linearity, selectivity, sensitivity and accuracy (precision and trueness).

Linearity. It was tested using the correlation coefficients $\left(\mathrm{R}^{2}\right)$ and the $\mathrm{P}$ values of the lack-of-fit test. $\mathrm{R}^{2}$ values ranged from 99.6 to $99.9 \%$ for the LC-FD method, from 99.2 to $99.5 \%$ for the LC-MS method and from 99.1 to $99.7 \%$ for the LC-MS/MS method. $P_{\text {lof }}$ values were higher than $5 \%$ in all cases. These facts indicate a good linearity within the stated ranges.

Selectivity. The specificity of the three methods was determined by comparing the chromatograms of blank with the corresponding spiked pig muscle samples. No interferences from endogenous substances were observed at the retention time of the analytes.

Sensitivity. The limits of detection (LOD) and quantification (LOQ) were calculated according with the IUPAC criterion [14] and the obtained values are shown in Table 1.

Accuracy (precision and trueness). To evaluate the overall precision of the methods, intra- and inter-day precision (as relative standard deviation, RSD) were estimated at three different concentrations for each compound (25.0, 50.0 and $100.0 \mathrm{ng}$ $\mathrm{g}^{-1}$ for LC-MS and LC-MS/MS, and 10.0, 20.0 and $40.0 \mathrm{ng} \mathrm{g}^{-1}$ for LC-FD). In the LCMS and the LC-MS/MS assess, five pig muscle samples were spiked, extracted and analyzed; in the LC-FD assess three spiked samples were extracted and analyzed in duplicate. The procedure was repeated three times on the same day to evaluate intra-day variability and on three consecutive days to determine inter-day variability. Trueness was evaluated by determining the recovery of known amounts of the tested compounds in pig muscle samples. Samples were analyzed using the three methods and the concentration of each compound was determined by interpolation in the standard calibration curve within the linear dynamic range and compared to the amount of analytes previously added to the samples. The results of precision and trueness, summarized in Table 1, fulfill the requirements defined by the EU legislation [7]. 
Application of the methods

212 Six different pig muscle samples purchased in different markets in the area of Granada

213 (Spain), were extracted, cleaned up and analyzed according to the three methods, in

214 order to prove the presence or not of quinolones in these tissues destined to human

215 consumption. The results obtained with the three methods were similar and showed that

216 one of the analyzed samples contain residues of MAR and OXO. The found

217 concentration of MAR was $62.0 \mathrm{ng} \mathrm{g}^{-1}$ and of OXO $20.0 \mathrm{ng} \mathrm{g}^{-1}$. Both values are lower

218 than the MRL established by the EU for these compounds. RSDs from the mean of the

219 values obtained with the three methods are 2.8\% for MAR and 3.6\% for OXO. Figures

2201,2 and 3 show the chromatograms of the positive sample using LC-FD, LC-MS (SIM

221 mode) and LC-MS/MS (MRM mode).

\section{Figure 1, 2 and 3}

Comparison of methods

All methods have a good linearity within the stated ranges, especially the LC-FD method that has the highest values of $\mathrm{R}^{2}$ in all cases. In relation to the selectivity, the identification of compounds in LC-FD is based on almost exclusively in its retention time; as well the compound must be fluorescent at particular wavelengths ( $\left.\lambda_{\text {exc }}, \lambda_{\text {em }}\right)$. In the case of LC-MS each compound is identified by its retention time and it characteristic $\mathrm{m} / \mathrm{z}$ (molecular ion, generally $\mathrm{M}+\mathrm{H}^{+}$). On the other hand, in LC-MS/MS as well as the retention time, the compounds are identified by two characteristic ions; the first one is used for quantification and the second for confirmation. In this technique, the ratio between quantification and confirmation ions is also used for the unequivocal identification of compounds. Therefore the LC-MS/MS method is the most appropriate from the point of view of selectivity. Related to sensitivity, the lowest LOD and LOQ were obtained when the LC-MS/MS method was used. The LODs were between 0.2 and $0.3 \mathrm{ng} \mathrm{g}^{-1}$ for the LC-MS/MS method; between 0.3 and $1.8 \mathrm{ng} \mathrm{g}^{-1}$ for the LC-MS method and between 0.1 and $2.1 \mathrm{ng} \mathrm{g}^{-1}$ for the LC-FD method. In all cases, these values are below of the MRL established by the EU in the Commission

242 Regulation 37/2010 amending Annexes I to IV to Council Regulation (EEC) No $2432377 / 90$ on pharmacologically active substances and their classification regarding 
maximum residue limits in foodstuffs of animal origin. However, the values obtained using the LC-MS/MS method are from 1.5 to 6 times lower than those obtained using the LC-MS method and from 2 up to 10 times lower than the ones obtained using the LC-FD method, except for DAN whose LOD and LOQ are lower using the LC-FD method. Therefore, the LC-MS/MS method is again the best in terms of sensitivity. In terms of accuracy, intra-day and inter-day precision of the methods were lower than $15 \%$ and this is within the acceptable limits proposed by the guidelines for bioanalytical method validation $(\leq 20 \%)$. In all cases RSD values for the LC-FD $(2-4 \%)$ method were lower than those obtained for the LC-MS (5-14\%) and LC-MS/MS (5-12\%) methods. Finally, recoveries were higher than $77 \%$ in all cases with the three methods. The best results were obtained when LC-MS/MS was used as analytical technique, except for oxolinic acid whose recovery is higher using the LC-FD method.

\section{Conclusions}

In this work, three procedures which allow the extraction, identification and quantification of the quinolones regulated by the EU in pig muscle samples have been compared. The methods include an extraction of the quinolones from the tissue, a clean-up step by SPE and separation and determination by LC-MS, LC-MS/MS and LC-FD detection. The LOD and LOQ of the three methods are much lower than the MRLs fixed by European Union. The lowest values were obtained when the LCMS/MS method was used. Comparable values of recoveries were obtained for the three methods and the best results of precision in terms of RSD were obtained for the LC-FD method. Therefore, the LC-FD method and the LC-MS/MS method are the ones with the best quality parameters. However, MS/MS have the important advantage of allowing the possibility of confirming (selectivity) the presence of these compounds by means of fragment abundance ratios at rather low concentration levels.

It could be concluded that because of its low cost, easier handling and good quality parameters the LC-FD method would be a good option for the routine analysis of quinolones in pig muscle samples and if a positive sample were found, the LCMS/MS method should be used to confirm and ensure the result. 


\section{Acknowledgements}

278

279 The authors are indebted to all participants, without whom this work would not have

280 been possible. This study was supported by the Spanish Ministry of Science and

281 Innovation (Project N ${ }^{0}$ CTQ2010-19044) and by the regional Government of Andalusia 282 (Project N ${ }^{\circ}$ P09-CTS-4470).

283 


\section{References}

1. Ternes TA, Joss A, Siegrist H (2004) Environ Sci Technol 38:392A-399A

2. Sukul P, Spiteller M (2007) Rev Environ Contam Toxicol 191:131-162

3. Andriole VT (2005) Clin Infect Dis 41:113-119

4. Ramos M, Aranda A, García E, Reuvers T, Hooghuis H (2003) J Chromatogr B Analyt Technol Biomed Life Sci 789:373-381

5. Martínez M, McDermott P, Walker R (2006) Vet J 172:10-28

6. Council Directive 96/23/EC (1996) Off J Eur Commun L125 10-18

7. Commission Decision 2002/657/EC (2002) Off J Eur Commun L221 8-62

8. Council Regulation (EEC) $\mathrm{N}^{\mathrm{o}} 2377 / 90$ of 26 June (1990) Off J Eur Commun L224 $1-8$

9. Commission Regulation (EU) $N^{\circ} 37 / 2010$ of 22 December (2009) Off J Eur Commun L15 1-72. http://europa.eu

10. Bailac S, Ballesteros O, Jiménez-Lozano E, Barrón D, Sanz-Nebot V, Navalón A, Vílchez JL, Barbosa J (2004) J Chromatogr A 1029:145-151

11. Hassouan MK, Ballesteros O, Zafra A, Vílchez JL, Navalón A (2007) J Chromatogr B 859:282-288

12. Hermo MP, Barrón D, Barbosa J (2006) J Chromatogr A 1104:132-139

13. Analytical Methods Committee (1994) Analyst 119:2363-2366

14. US Department of Health and Human Services (2001) Bioanalytical Method Validation

15. Currie LA (1999) Anal Chim Acta 391:105-126 


\section{Figure Captions}

Fig. 1 LC-FD. Contaminated sample with MAR and OXO. Concentration: MAR, 64 $\mathrm{ng} \mathrm{g}^{-1}$; OXO, $24 \mathrm{ng} \mathrm{g}^{-1}$; and IS, $20 \mathrm{ng} \mathrm{g}^{-1}$.

Fig. 2 LC-MS in SIM mode. Contaminated sample with MAR and OXO. Concentration: MAR, $59 \mathrm{ng} \mathrm{g}^{-1}$; OXO, $18 \mathrm{ng} \mathrm{g}^{-1}$; and IS, $400 \mathrm{ng} \mathrm{g}^{-1}$.

Fig. 3 LC-MS/MS in MRM mode. (A) Contaminated sample with MAR and OXO. (B) Confirmatory chromatograms of MAR and OXO. Concentration: MAR, $62 \mathrm{ng} \mathrm{g}^{-}$ ${ }^{1}$; OXO, $18 \mathrm{ng} \mathrm{g}^{-1}$; and IS, $400 \mathrm{ng} \mathrm{g}^{-1}$. 


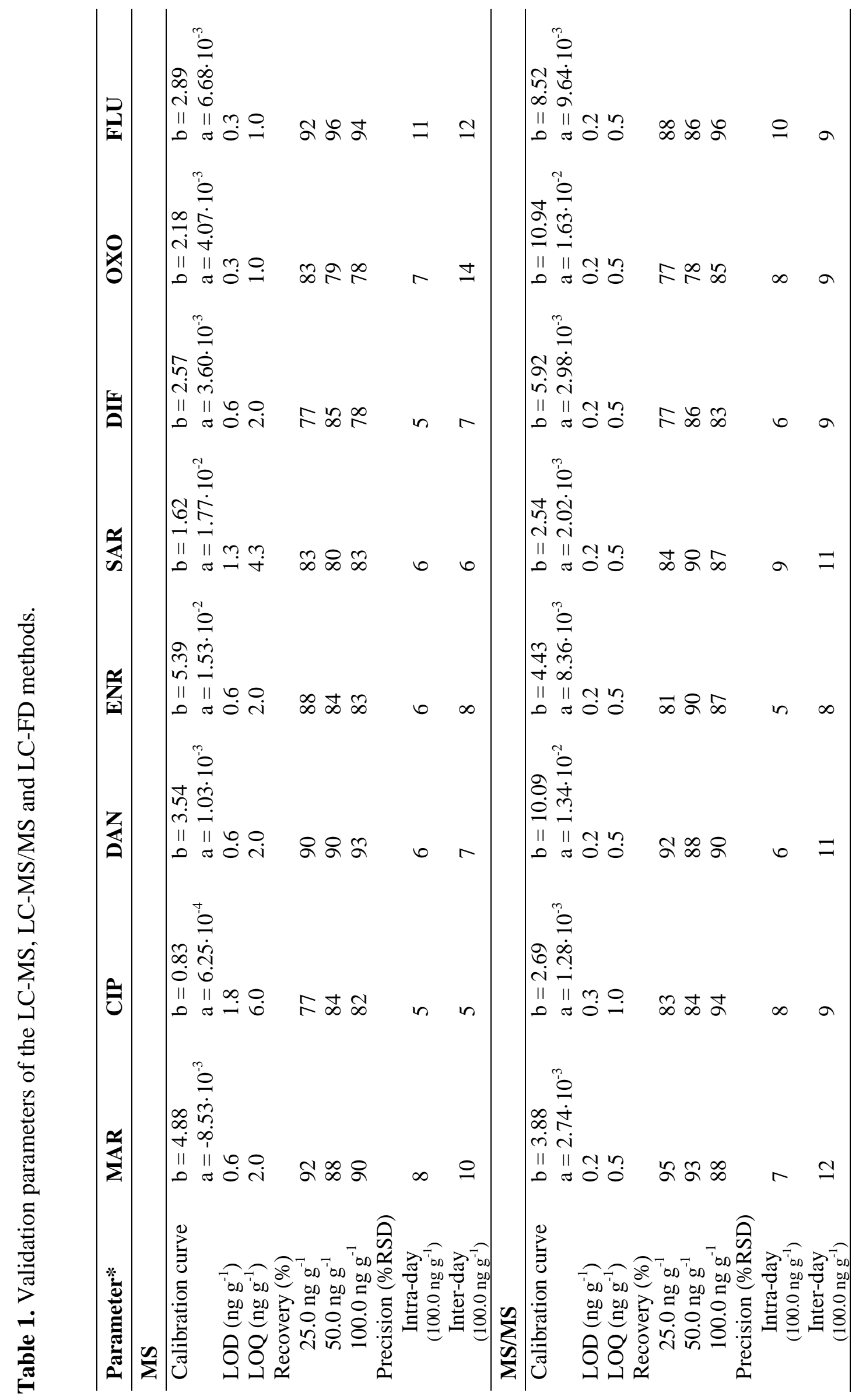




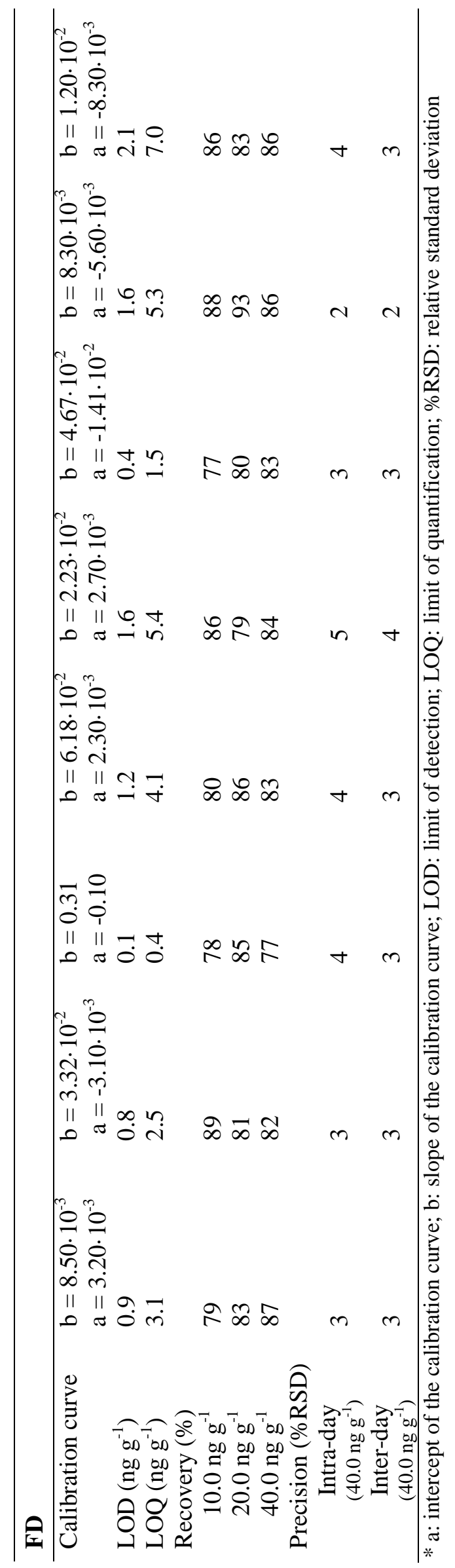




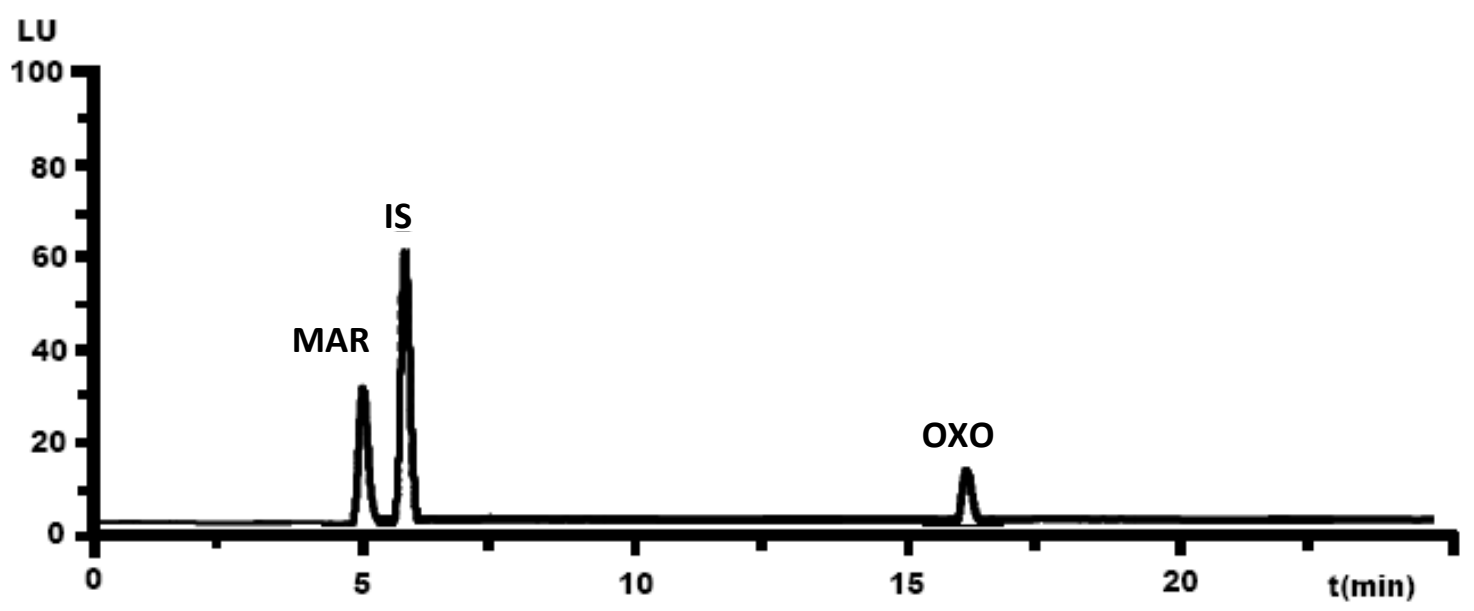

Figure 1 

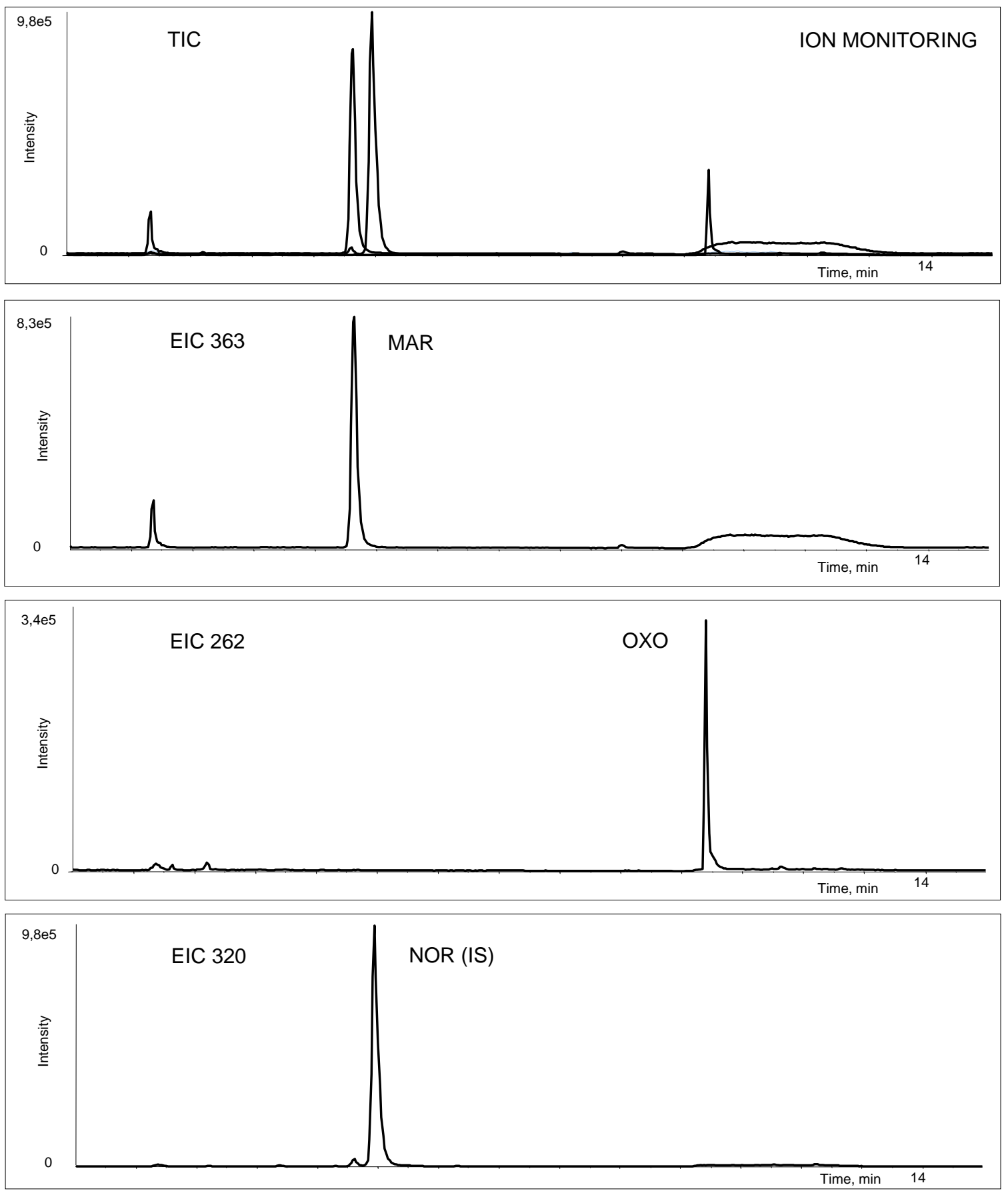

Figure 2 

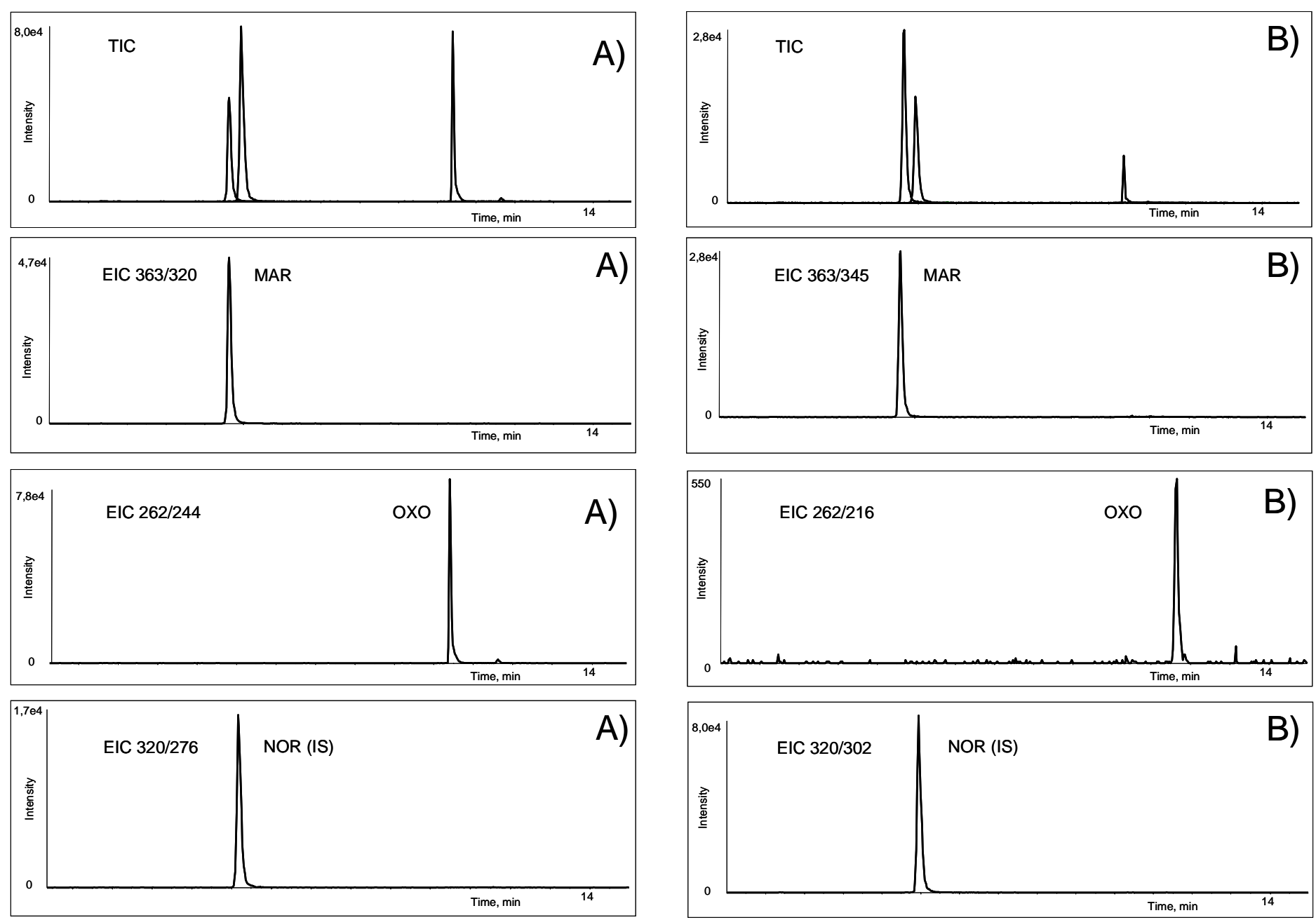

Figure 3 